OPEN ACCESS

Edited by:
Zongxin Ling,
Zhejiang University, China

Reviewed by:

Jian Song,

University Hospital Münster, Germany Yanli Zhang,

University of Cambridge, United Kingdom

Jiarui Zhang,

Boston University, United States

${ }^{*}$ Correspondence: Xiaoping Wan

wanxiaoping@tongji.edu.cn

Yiran Li

yiran_eric@126.com

${ }^{t}$ These authors have contributed equally to this work

Specialty section:

This article was submitted to Microbiome in Health and Disease,

a section of the journal

Frontiers in Cellular and

Infection Microbiology

Received: 28 July 2021 Accepted: 06 September 2021 Published: 21 September 2021

Citation:

Li C, Gu Y, He Q, Huang J, Song Y, Wan $X$ and Li Y (2021) Integrated

Analysis of Microbiome and Transcriptome Data Reveals the Interplay Between Commensal Bacteria and Fibrin Degradation in Endometrial Cancer. Front. Cell. Infect. Microbiol. 11:748558. doi: 10.3389/fcimb.2021.748558

\section{Integrated Analysis of Microbiome and Transcriptome Data Reveals the Interplay Between Commensal Bacteria and Fibrin Degradation in Endometrial Cancer}

\author{
Chao $\mathrm{Li}^{1 \dagger}$, Ye Gu${ }^{2 \dagger}$, Qizhi He ${ }^{3}$, Jian Huang ${ }^{1}$, Yunfeng Song ${ }^{1}$, Xiaoping Wan ${ }^{2 *}$ \\ and Yiran $\mathrm{Li}^{2 *}$ \\ 1 Shanghai Key Laboratory of Maternal Fetal Medicine, Shanghai First Maternity and Infant Hospital, School of Medicine, \\ Tongji University, Shanghai, China, ${ }^{2}$ Department of Gynecology, Shanghai First Maternity and Infant Hospital, Tongji \\ University School of Medicine, Shanghai, China, ${ }^{3}$ Department of Pathology, Shanghai First Maternity and Infant Hospital, \\ Tongji University School of Medicine, Shanghai, China
}

The gut-uterus axis plays a pivotal role in the pathogenesis of endometrial cancer (EC). However, the correlations between the endometrial microbiome and endometrial tumor transcriptome in patients with $\mathrm{EC}$ and the impact of the endometrial microbiota on hematological indicators have not been thoroughly clarified. In this prospective study, endometrial tissue samples collected from EC patients $(n=30)$ and healthy volunteers $(n=10)$ were subjected to $16 \mathrm{~S} r R N A$ sequencing of the microbiome. The 30 paired tumor and adjacent nontumor endometrial tissues from the EC group were subjected to RNAseq. We found that Pelomonas and Prevotella were enriched in the EC group with a high tumor burden. By integrating the microbiome and hematological indicators, a correlation was observed between Prevotella and elevated serum D-dimer (DD) and fibrin degradation products (FDPs). Further transcriptome analysis identified 8 robust associations between Prevotella and fibrin degradation-related genes expressed within ECs. Finally, the microbial marker of Prevotella along with DD and FDPs showed a high potential to predict the onset of EC (AUC = 0.86). Our results suggest that the increasing abundance of Prevotella in endometrial tissue combined with high serum DD and FDP contents may be important factors associated with tumor burden. The microbe-associated transcripts of host tumors can partly explain how Prevotella promotes DD and FDPs.

Keywords: endometrial cancer, microbiome, pyrosequencing, transcriptome, biomarker

\section{INTRODUCTION}

Endometrial cancer (EC) is the most common female reproductive tract malignancy in developed countries and shows an increasing incidence (Bray et al., 2018). Importantly, the incidence of EC is drastically rising in high-income countries (Jonusiene and Sasnauskiene, 2021). Although several mechanistic events accompanying host genetic alterations and hereditary factors have been shown 
to play important roles in endometrial carcinogenesis, they can only explain 10-20\% of cases (Walther-António et al., 2016; Kuźmycz and Staczek, 2020). Efforts to identify the cause of the remaining $80-90 \%$ of cases have led to studies on a number of environmental factors, including hormones, obesity, inflammation, and menopausal status, which are major risk factors for the development of type I EC (Beral et al., 2005; Morice et al., 2016). There is an urgent need to identify previously unrecognized mechanisms for the diagnosis and therapeutic management of EC. However, the underlying mechanisms involved in the occurrence and development of EC are far from being explored.

Available evidence has shown the potential involvement of microbiota in the development of different types of human cancer, including endometrial cancer (Lu et al., 2021). Of interest, several cross-sectional studies have noted a link between microbiota composition and EC (Walther-António et al., 2016; Walsh et al., 2019; Lu et al., 2021). Therefore, endometrial microbiota could be implicated as an environmental influence that contributes to the progression of EC. Nonetheless, the profile of the endometrial microbial community and its function in endometrial carcinogenesis remain unclear. Only one study has explored whether the increased abundance of Micrococcus is positively correlated with IL-6 and IL-17, which are involved in the proinflammatory response in EC (Lu et al., 2021). Host genes are known as important regulators connecting microbiota to cancer (Huang et al., 2020). Microbiota can alter the expression of host genes and exert a tumor stimulative role through multiple mechanisms, such as by modifying signaling proteins and modulating subsequent transcriptional responses (O'Keefe, 2016). However, the interplay between the endometrial microbial community and host transcriptome and their roles in EC development have not been addressed.

In this prospective study, we investigated the endometrial microbiota composition in a cohort of 10 healthy controls (HCs) and 30 EC patients using high pyrosequencing of barcoded $16 \mathrm{~S}$ rRNA genes (V3-V4). The transcriptome of the paired tumor and adjacent nontumor endometrial tissues from the $30 \mathrm{EC}$ patients was also determined. By the simultaneous integrated analysis of the endometrial tumor transcriptome, endometrial microbiome, and hematological indicators, we aimed to gain better insights into the nature of the gut-transcriptome-uterus axis in EC patients.

\section{METHODS}

\section{Participant Information}

This study was conducted at Shanghai First Maternity and Infant Hospital affiliated with Tongji University from March 2018 to July 2020. The inclusion criteria of the participants were as follows: (a) women aged between 40 and 69 years; and (b) subjects undergoing hysterectomy by any standard surgical approach for any possible benign disease and at stage I endometrial cancer (EC). The exclusion criteria were as follows: (a) pregnant women and nursing mothers; (b) women who used antibiotics within 3 months; (c) history of genital tract infection or medication within 3 months; and (d) patients receiving preoperative chemotherapy or radiotherapy. A total of approximately 120 subjects were recruited, and after filtering with the inclusion and exclusion criteria, 40 women, including 30 endometrial cancer (EC) patients and 10 healthy controls (HCs), were finally enrolled (Table S1).

Endometrial tissue samples were acquired from these 40 women, who had undergone a hysterectomy. After removing the corrupted and erosive tissues of the surface, the residual tissues were macrodissected. The whole process was performed by an experienced gynecologist under strict aseptic procedures. The samples were divided into three equal parts (one for $16 \mathrm{~S}$ rRNA sequencing, one for RNA-seq, and one for subsequent experimental verification), snap frozen and stored at $-80^{\circ} \mathrm{C}$.

\section{Bacterial DNA Extraction and Amplification}

Bacterial DNA was isolated from the endometrial samples using a DNeasy PowerSoil kit (Qiagen, Hilden, Germany) following the manufacturer's instructions. DNA concentration and integrity were measured by a NanoDrop 2000 spectrophotometer (Thermo Fisher Scientific, Waltham, MA, USA) and agarose gel electrophoresis, respectively. PCR amplification of the V3-V4 hypervariable regions of the bacterial $16 \mathrm{~S}$ rRNA gene was carried out in a $25 \mu \mathrm{L}$ reaction using universal primer pairs (343F: 5' - TACGGRAGGCAGCAG-3'; 798R: 5' AGGGTATCTAATCCT-3') (Chen et al., 2018). The reverse primer contained a sample barcode, and both primers were connected with an Illumina sequencing adapter.

\section{Library Construction and Sequencing}

The amplicon quality was visualized using gel electrophoresis. The PCR products were purified with Agencourt AMPure XP beads (Beckman Coulter Co., USA) and quantified using a Qubit dsDNA assay kit. The concentrations were then adjusted for sequencing. Sequencing was performed on an Illumina MiSeq with two paired-end read cycles of 300 bases each (Illumina Inc., San Diego, CA; OE Biotech Company; Shanghai, China). The raw data for the Illumina reads from 40 endometrial samples were uploaded to the Sequence Read Archive (SRA) database.

\section{Bioinformatic Analysis}

Paired-end raw sequences were preprocessed using Trimmomatic software (version 0.36) (Bolger et al., 2014) to detect and cut off ambiguous bases $(\mathrm{N})$, and this software was also used to cut off low-quality sequences, with an average quality score below 20 using a sliding window trimming approach. After trimming, paired-end reads were assembled using FLASH software (version 1.2.11) (Reyon et al., 2012). Sequences were further denoised using QIIME software (version 1.8.0) (Caporaso et al., 2010). Then, reads with chimeras were detected and removed using VSEARCH (version 2.4.2) (Rognes et al., 2016). Clean reads were subjected to primer sequence removal and clustering to generate operational taxonomic units (OTUs) using VSEARCH software, with a 97\% similarity cutoff (Rognes et al., 2016). The representative read of each OTU was selected using the QIIME package. All representative reads were annotated and blasted 
against the Silva database (Version 123) using the RDP classifier (confidence threshold was 70\%) (Wang et al., 2007). The microbial diversity in the endometrial samples was estimated using the alpha diversity, which includes the Chaol index, observed species index, Simpson index, and Shannon index (Yang et al., 2019). The unweighted UniFrac distance matrix performed by QIIME software (version 1.8.0) was used for nonmetric multidimensional scaling (NMDS) plots. A Venn diagram was generated to visualize the shared and unique genera among groups regardless of their relative abundance using the R package "VennDiagram" (Zaura et al., 2009). The LEfSe (linear discriminant analysis effect size) method was used to detect differentially abundant taxa across groups using the default parameters (Segata et al., 2011). 16S rRNA gene amplicon sequencing and analysis were conducted by OE Biotech Co., Ltd. (Shanghai, China).

\section{RNA Extraction, RNA-Seq, and Data Analysis}

Total RNA was extracted from frozen endometrial tissues by using mirVana ${ }^{\mathrm{TM}}$ miRNA Isolation Kit (catalog no., AM1561, Ambion ${ }^{\circledR}$ ), and cDNA libraries were sequenced on the MGISeq2000 Platform (MGI, Shenzhen, China). Then, $150 \mathrm{bp}$ paired-end reads were generated. Approximately 49.35 million raw reads for each sample were generated. Raw data (raw reads) in fastq format were first processed using Trimmomatic software (Bolger et al., 2014), and the low-quality reads were removed to obtain clean reads. Then, approximately 48.57 million clean reads for each sample were retained for subsequent analyses.

The clean reads were mapped to the human genome (GRCh38) using HISAT2 (version 2.2.1.0) (Kim et al., 2015). The FPKM (Roberts et al., 2011) value for each gene was calculated using Cufflinks (version 2.2.1) (Trapnell et al., 2010), and the read counts of each gene were obtained by HTSeq-count (version 0.9.1) (Anders et al., 2015). A differential expression analysis was performed using the DESeq (2012) R package (version 3.2.0, R Foundation for Statistical Computing, Vienna, Austria). A p value $<0.05$ and fold change $>2$ or fold change $<0.5$ were set as the thresholds for significantly differential expression. A hierarchical cluster analysis of differentially expressed genes (DEGs) was performed to demonstrate the expression pattern of genes in different groups and samples. GO enrichment and KEGG pathway enrichment analyses of DEGs were performed using $\mathrm{R}$ based on the hypergeometric distribution.

\section{Correlation Between the Genus and Clinical Hematological Characteristics}

The Pearson correlation coefficient was calculated to evaluate the clinical characteristics that underwent significant changes (values of serum D-dimer (DD), fibrin degradation products (FDPs), cancer antigen (CA) 125, CA199, and total protein) and endometrial microbiota at the genus level between $\mathrm{HC}$ and $\mathrm{EC}$ women. To reduce the computational load and avoid contingency, the genera that presented an abundance value of $\leq 0.1 \%$ were excluded. The significance of each genus-hematological indicator pair was determined based on a $p$ value $<0.05$ and a false discovery rate $(\mathrm{FDR})<0.1$. Detailed scripts of the correlation calculations are provided in Table $\mathbf{S} 2$.

\section{Correlation Between Genera and Differentially Expressed Genes}

The Pearson correlation coefficient was calculated to measure the connections between genus abundance and differential gene expression level for each genus-gene pair across all 30 patients. To reduce the computational load and avoid contingency, the genera that presented an abundance value of $\leq 0.1 \%$ were excluded. The significance of each genus-gene pair was determined based on a $p$ value $<0.05$ and FDR $<0.1$. Independent Student's $t$ test was applied to evaluate the difference in $\log _{2} \mathrm{FC}$ values calculated by DESeq between the HC and EC groups. Detailed scripts of the correlation calculations are provided in Table S3.

\section{Immunofluorescence Staining}

Paraffin-embedded endometrial tissue sections were treated as previously described (Li et al., 2019). After retrieval of antigenic binding sites, the sections were permeabilized with $0.2 \%$ Triton $\mathrm{X}-100$ for $10 \mathrm{~min}$, and then blocked with $5 \%$ bovine serum albumin for up to $1.5 \mathrm{~h}$ at room temperature, and incubated with appropriate concentrations of primary antibodies against PRSS33 (Abmart, Shanghai, China, TD4461M, 1:200 dilution), CPB2 (Bioss Antibodies, Beijing, China, BS-7547R, 1:200 dilution), and XBP1 (Abcam, London, UK, ab37152, $20 \mu \mathrm{g} /$ $\mathrm{mL}$ ) overnight at $4^{\circ} \mathrm{C}$. Then, the sections were incubated with Alexa Fluor ${ }^{\circledR}$ 488/594/647 conjugated goat anti-rabbit IgG (Abcam, London, UK, 1:200 dilution) secondary antibodies for $1 \mathrm{~h}$. Cell nuclei was stained with DAPI. The stained sections were observed under a fluorescence confocal microscope.

\section{Statistical Analysis}

Fundamental statistical analyses were performed using SPSS software (version 19.0, IBM SPSS Inc., Chicago, IL, USA), GraphPad Prism 7 software (GraphPad software, Inc., San Diego, California, USA) and $\mathrm{R}$ version 3.2.0. Categorical variables were expressed as numbers (\%). For normally distributed variables, the Student's $t$ test was used; otherwise, the Wilcoxon rank sum test was used. Pearson's rank correlation analysis was conducted to calculate the correlation between genera, between genera and clinical characteristics, or between genera and DEGs. All statistical tests were two-tailed, and the $p$ value was adjusted by the Benjamini-Hochberg correction. A $p$ value $<0.05$ was considered statistically significant.

\section{RESULTS}

\section{Summary of Clinical Characteristics}

All 30 endometrial cancer (EC) patients and 10 healthy control (HC) women were Han Chinese individuals from Shanghai. All diagnoses were performed based on the final surgical pathology. The demographics and other clinicopathological characteristics are shown in Table 1. The control participants were generally matched with the case participants by age and BMI, suggesting that there were no established confounding factors that might 
influence group discrimination prior to sample collection. There was a significant increase in serum DD $(p=0.0122)$, FDP $(p=0.0003)$, CA125 $(p=0.0094)$ and CA199 $(p=0.0261)$ levels and a significant decrease in serum total protein in EC patients $(p=0.0441)$ (Table S1).

\section{Decreased Bacterial Diversity in Endometrial Microbiota Associated With EC}

We analyzed a total of 40 endometrial samples based on the pyrosequencing of barcoded 16S rRNA genes (V3-V4) and finally obtained 2,728,218 qualified sequences (median $=68,151$ ) and 9,278 OTUs (Tables S4, S5). The rarefaction curves indicated that the estimated richness of OTUs almost reached saturation in the HC $(n=10)$ and EC groups $(n=30)$ (Figure 1A). Moreover, the rank abundance curves indicated decreased richness in the EC patients compared with the HCs (Figure S1).

To estimate the differences in bacterial diversity between the two groups, the sequences were aligned to estimate alpha diversity and beta diversity (Table S6). Significant differences between the EC and HC groups were observed in the Chaol (2247.72 \pm 151.01 versus $2476.16 \pm 142.47, p<0.001)$, observed species $(1321.61 \pm 114.95$ versus $1420.98 \pm 117.80, p=0.0212)$ and Simpson (0.989 \pm 0.007 versus $0.993 \pm 0.001, p=0.0053$ ) indexes (Figures 1B-D), whereas significant differences between these groups were not observed for the Shannon index $(7.99 \pm$ 0.41 versus $8.18 \pm 0.26, p=0.0606$ ) (Figure S2). A Venn diagram showed that 100 out of 147 genera were shared between the EC and HC groups (Figure 1E). Notably, 20 out of 147 genera were

TABLE 1 | Clinical characteristics summary of all enrolled women.

\begin{tabular}{|c|c|c|c|}
\hline Category & $\begin{array}{l}\text { Healthy control } \\
\qquad(n=10)\end{array}$ & $\begin{array}{l}\text { Endometrial } \\
\text { cancer group } \\
(n=30)\end{array}$ & $P$ value \\
\hline \multicolumn{4}{|l|}{ Clinical and pathological feature } \\
\hline Age (year) & $53.1 \pm 6.67^{\mathrm{a}}$ & $56.4 \pm 7.89^{a}$ & 0.1042 \\
\hline Body mass index $\left(\mathrm{kg} / \mathrm{m}^{2}\right)$ & $22.89 \pm 2.15^{a}$ & $22.70 \pm 1.66^{\mathrm{a}}$ & 0.4072 \\
\hline Histology & & & - \\
\hline Endometrioid & - & $22(73.3 \%)^{b}$ & \\
\hline Serous & - & $8(26.7 \%)^{b}$ & \\
\hline FIGO stage & & & - \\
\hline$|-| \mid$ & - & $30(100 \%)^{b}$ & \\
\hline III-IV & - & $0(0 \%)^{b}$ & \\
\hline \multicolumn{4}{|l|}{ Hematologic biomarker ${ }^{a}$} \\
\hline Total protein (64.0-83.0 g/L) & $74.49 \pm 1.73$ & $72.42 \pm 5.76$ & 0.0441 \\
\hline Albumin $(35.0-55.0 \mathrm{~g} / \mathrm{L})$ & $45.86 \pm 2.32$ & $44.73 \pm 4.02$ & 0.1422 \\
\hline Globulin (20.0-35.0 g/L) & $28.63 \pm 2.30$ & $27.69 \pm 3.72$ & 0.1771 \\
\hline Prothrombin time (11-13 s) & $11.17 \pm 0.74$ & $11.0 \pm 0.59$ & 0.26 \\
\hline Fibrinogen (2.0-4.0 g/L) & $2.43 \pm 0.48$ & $2.65 \pm 0.61$ & 0.13 \\
\hline D-dimer (0-0.5 mg/L) & $0.19 \pm 0.07$ & $0.42 \pm 0.52$ & 0.0122 \\
\hline $\begin{array}{l}\text { Fibrin degradation products } \\
(0-5.0 \mathrm{mg} / \mathrm{L})\end{array}$ & $0.96 \pm 0.45$ & $1.91 \pm 1.70$ & 0.0045 \\
\hline Cancer antigen 125 (0-35.0 U/mL) & $8.20 \pm 5.34$ & $29.37 \pm 45.87$ & 0.0094 \\
\hline Cancer antigen 199 (0-37.0 U/mL) & $5.17 \pm 2.79$ & $46.43 \pm 111.49$ & 0.0261 \\
\hline Alpha fetoprotein (0-20.0 mg/L) & $2.63 \pm 0.94$ & $3.13 \pm 1.30$ & 0.1008 \\
\hline $\begin{array}{l}\text { Carcinoembryonic antigen } \\
(0-5.0 \mathrm{mg} / \mathrm{L})\end{array}$ & $1.64 \pm 0.82$ & $1.93 \pm 1.36$ & 0.2138 \\
\hline
\end{tabular}

${ }^{a}$ Values are given as mean $\pm S D$.

${ }^{b}$ Categorical variables were expressed as numbers (\%). -, not exist. unique to the EC group. For the beta diversity, the NMDS plots evaluated by the unweighted UniFrac distance matrix exhibited a separation of the distribution of the two groups (Figure 1F). The results suggest that the diversity of endometrial microbiota could be strongly influenced by the tumor burden.

\section{Alterations in the Composition of Endometrial Microflora Associated With EC}

The bacterial distribution was assessed according to the relative abundance of different taxa. The five dominant phyla in the EC and HC groups were Bacteroidetes, Proteobacteria, Firmicutes, Actinobacteria, and Cyanobacteria, which accounted for $92.17 \%$ and $92.67 \%$ of the total OTUs, respectively (Figure $\mathbf{2 A}$ ). The compositions of the bacterial community (top 15) at the genus level are shown in Figure 2B. To provide insight into the differences in endometrial microflora between the two groups, the Wilcoxon rank sum test was performed at both the phylum and genus levels (Tables S7, S8). Compared with the HC group, Pelomonas, Prevotella, Nocardioides and Muribaculum were enriched in the EC group, whereas Oscillibacter exhibited the opposite trend (all $p<0.05$, Figure $2 \mathrm{C}$ ).

Next, the LEfSe method was used to discover highdimensional biomarkers. Of the above five genera, Pelomonas and Prevotella were significantly overrepresented [LDA scores $\left(\log _{10}\right)>3$ ] in the endometrium of EC patients (Figure 2D). The relative abundances of the two genera were further subjected to a cluster analysis, and the results suggested that Pelomonas and Prevotella were abundant in the EC group (Figure 2E). Sankey diagrams were also generated to visualize the major proportion of taxa (phylum and genus) between the HC and EC groups. In patients with a tumor burden, the proportion of Pelomonas and Prevotella gradually increased and became predominant, accompanied by changes in other bacteria (Figure 2F). Overall, these data suggest that the differentially abundant microbiota, including Pelomonas and Prevotella, were able to distinguish the microbiota between the HC volunteers and EC patients.

\section{Serum DD and FDPs Were Associated With Altered Microflora in EC}

For the intraindividual correlation analyses, we tested the associations between clinical characteristics (abnormal values of serum DD, FDPs, CA125, CA199, and total protein) and endometrial microbiota. Pearson's correlation-based analysis indicated that the three genera Prevotella, Acinetobacter and Brevundimonas were remarkably associated with fibrin degradation (DD and FDPs), while meaningful results based on other clinical characteristics were not observed (Figure 3A, Table S2). Among the three genera, Prevotella microbes were correlated with the aforementioned different endometrial microbiota composition alterations in the $\mathrm{HC}$ and EC groups (Figures 3B, C). Therefore, Prevotella were selected for further study.

\section{Global Overview of Endometrial Tumor Transcriptome in EC}

Since the EC patients demonstrated signature microbiota associated with clinical characteristics (tumor burden), we hypothesized that 


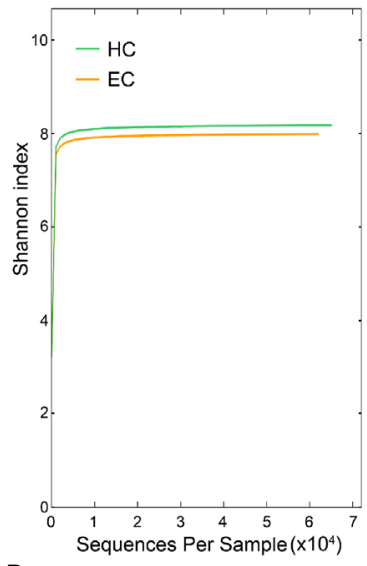

$$
\text { D }
$$

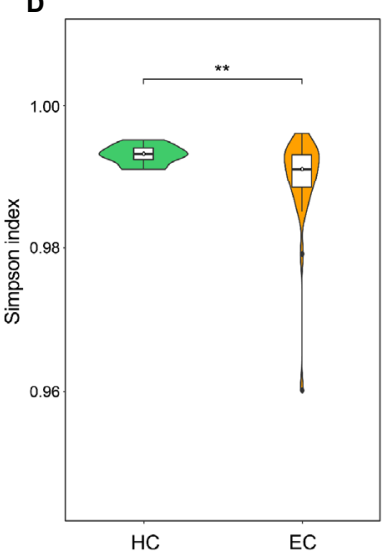

B

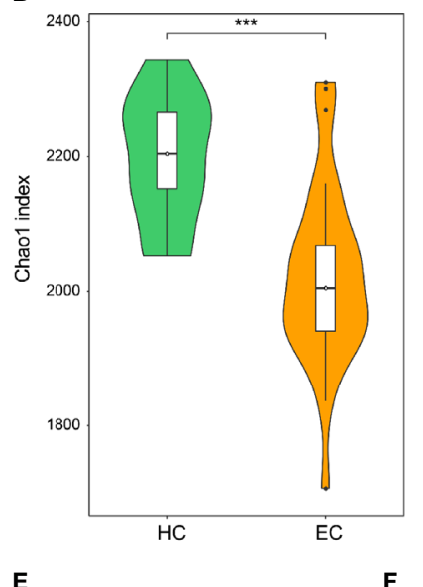

C

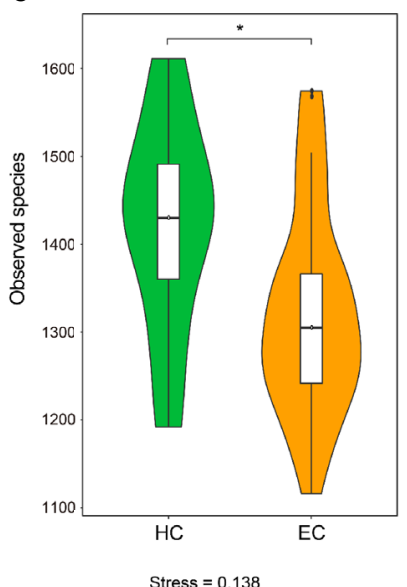

E

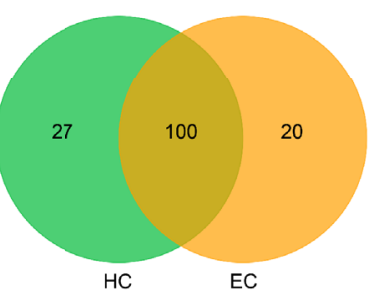

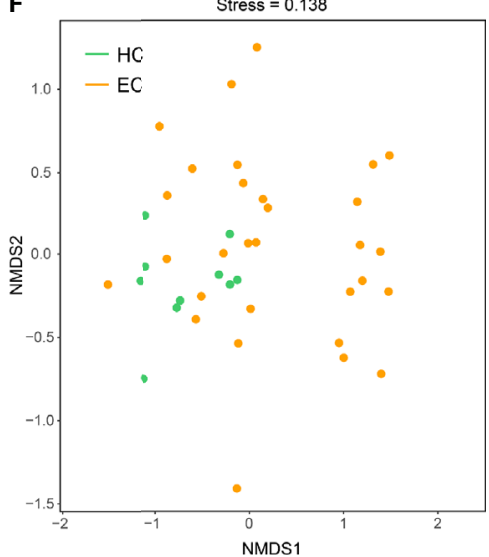

FIGURE 1 | Endometrial microbial diversity of HC and EC participants. (A) Shannon-Wiener rarefaction curve of HC and EC groups. (B-D) $\alpha$-Diversity comparison between different disease states in the endometrial microbiome. (B), Chao1 index; (C), observed species; and (D), Simpson index. ${ }^{\star} p<0.05$, ${ }^{* *} p<0.01$, ${ }^{\star \star \star} p<0.001$, Wilcoxon rank sum test. (E) Venn diagram illustrating the shared and unique genera between HC and EC. (F) Beta diversity evaluated using NMDS with an unweighted UniFrac distance matrix. HC, healthy control; EC, endometrial cancer.

changes in the transcriptome of endometrial tumorigenesis may be correlated with endometrial microbiota. Thus, we performed a transcriptome analysis of the paired tumor and adjacent nontumor endometrial tissues from 30 EC patients. Based on our definition, we identified a total of 3,911 differentially expressed genes (with 1,852 upregulated and 2,059 downregulated) among the 30 paired endometrial tissues by DESeq (Figure 4A). The genes whose $\log _{2} \mathrm{FC}$ values calculated by GFOLD were zero in $>90 \%$ of patients were excluded, thus leaving 3,410 genes for further investigation of the correlations with Prevotella.

\section{Prevotella Promotes DD and FDPs by Influencing the Host Endometrial Transcriptome Profile}

Furthermore, Pearson's correlation-based analysis was performed to discover Prevotella-associated genes and to examine whether the levels of DD and FDPs could be partially influenced by these genes. Among the 3410 genes, 74 genus-gene pairs were formed with FDR $<0.1$ (Table S3). A total of 8 genes were identified to potentially affect the degradation of fibrin (peptidase activity, proteolysis, protein digestion and absorption, and cellular secretory pathway transcription factor) (Figure 4B). The pathway analysis confirmed that these 8 genes converged mainly on proteolysis-related pathways (Figure 4C). A subsequent correlation analysis showed three examples of Prevotella-gene pairs (Prevotella-serine protease 33 (PRSS33), Prevotella-carboxypeptidase B2 (CPB2), Prevotella-xbox binding protein 1 (XBP1)) (Figures 4D-F). The mammalian PRSS33 is primarily responsible for degrading fibrin into DD and FDPs within blood (Izem et al., 2021). CPB2 is involved in the clotting-fibrinolytic pathway, and abnormal expression of this protein has been linked to thromboembolism and cancer (Lip et al., 2002). XBP1 is a key transcription factor of the cellular secretory system, and it is often overexpressed in cancers, such as oral squamous cell carcinoma and hepatocellular carcinoma, and correlates with the clinical outcome (Stelloo et al., 2020). Multiplex immunofluorescence confirmed that PRSS33, CPB2 and XBP1 proteins were significantly highly expressed in the endometrial tumor tissues of patients with elevated DD and FDPs (Figure 4G), suggesting that Prevotella could partially promote DD and FDPs by influencing host gene expression. To further validate the other 2 
A

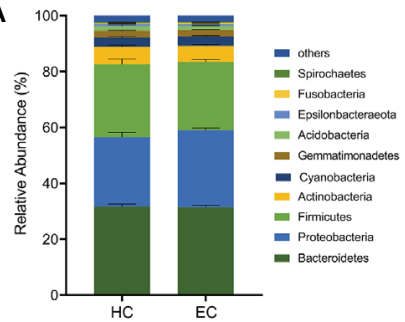

D

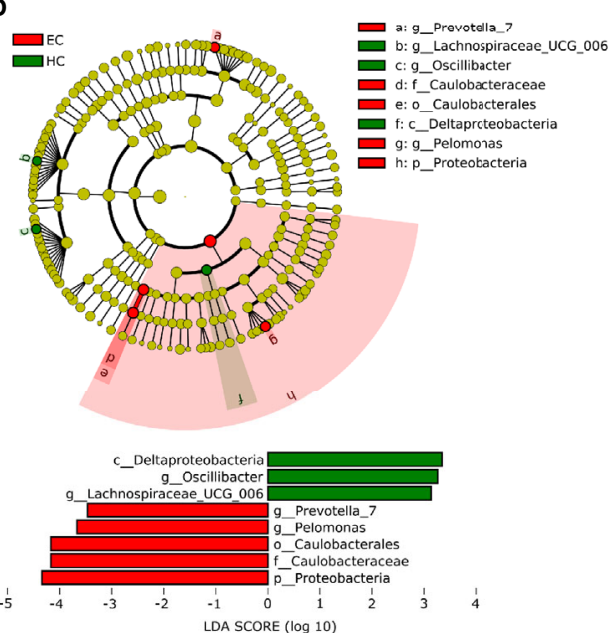

B

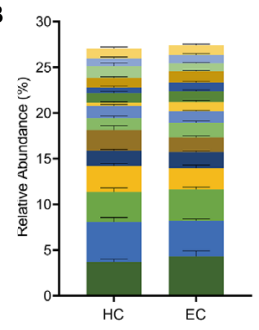

E
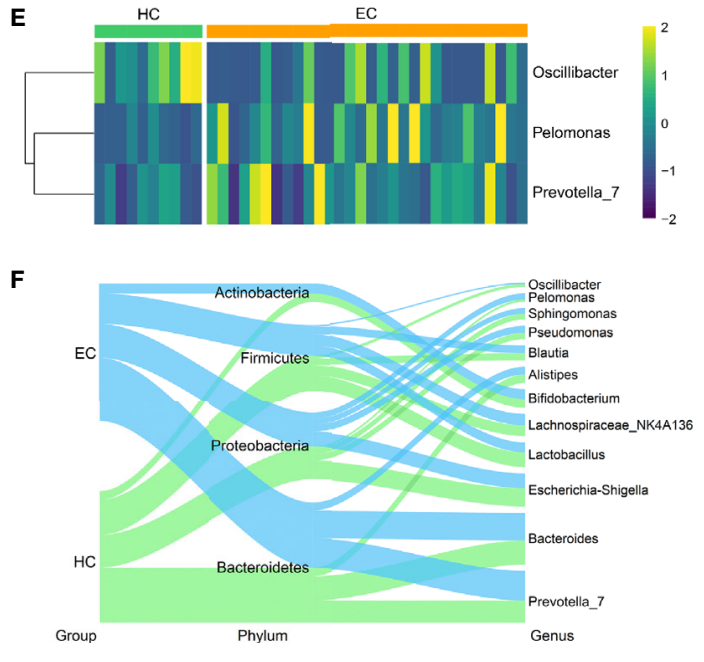

= Faccaibaaculum $=10$ Sptingomononas $=$ Bautia

- Faccaibacatorium

$=$ Psecdomonose

$=$ Alisipos

E- Btidobacterium

- Lachnospitraceae_NKaA 13

$=$ Escherchchla-Shigella

- Solanum_lorum

$=$ Prevolela

- Bacteroides

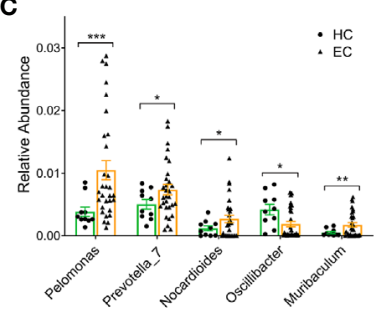

:

(1)

FIGURE 2 | Phylogenetic profiles of endometrial microbiota between the HC and EC groups. (A) Compositions of the bacterial community at the phylum level between HC and EC. (B) Compositions of the bacterial community (top 15) at the genus level between HC and EC. (C) Differential microbial community at the genus level in EC versus HC. Error bars are presented as the SD. (D) LEfSe analysis for comparing microbial variations in HC and EC at the genus level. LEfSe cladogram representing differentially abundant taxa $(p<0.05)$; LDA scores are calculated based on the LEfSe of differentially abundant taxa among groups, and only taxa with LDA scores of $>3$ are presented. (E) Distributions of Oscillibacter, Pelomonas, and Prevotella normalized by a Z-score between HC and EC. (F) Sankey analysis of $\mathrm{HC}$ and EC. ${ }^{\star} \mathrm{p}<0.05,{ }^{\star \star} \mathrm{p}<0.01,{ }^{\star \star \star} \mathrm{p}<0.001$.
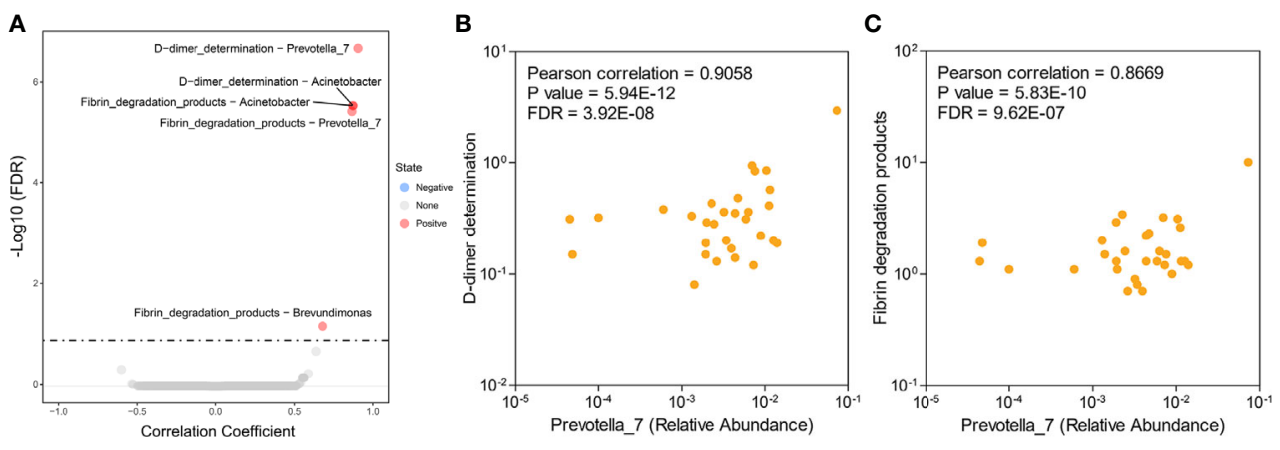

FIGURE 3 | Associations between endometrial microbiota and hematological indicators in patients with EC. (A) Differential level of the microbe-associated hematological indicator from 30 EC samples. (B, C) Scatter plots of two Prevotella-related hematological indicators: Prevotella-D-dimer (B) and Prevotella-fibrin degradation products (C). Each point represents a patient.

genes (KCNN4 and KLK11) under tumor burdens, DESeq was used to calculate the correlation between Prevotella and the KCNN4 and KLK11 genes (Figure S3). The results showed that these two genes satisfied the definition of differential genes between EC and adjacent nontumor endometrial tissues.

\section{Identification of Microbial Blood-Based Markers for Clinical Prediction}

According to the abovementioned analysis, we focused on Prevotella, DD and FDPs as potential biomarkers because they were abundant in the EC group. A receiver operating 
A

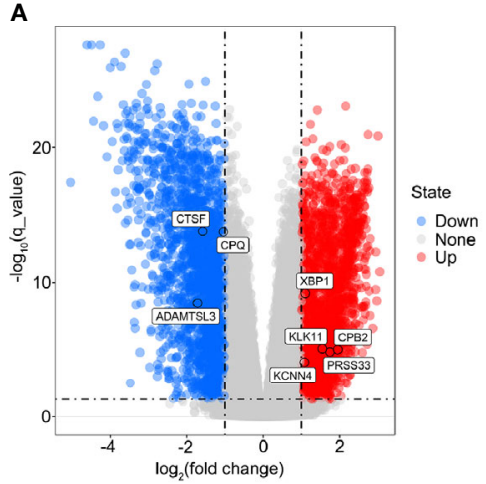

C

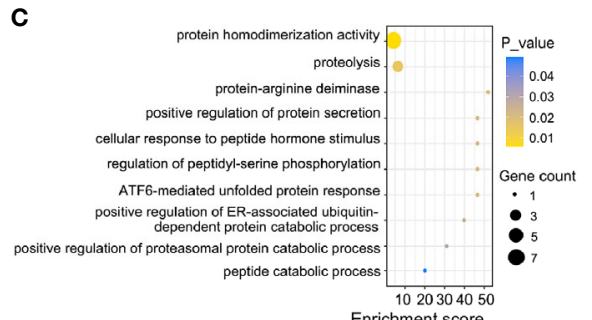

B

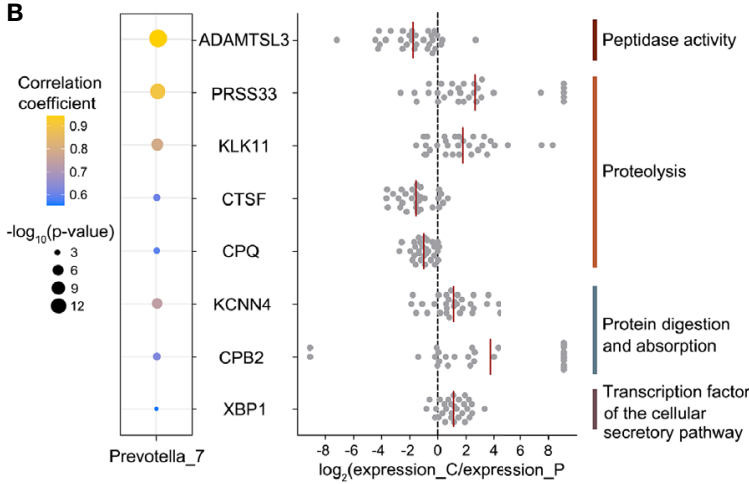

D

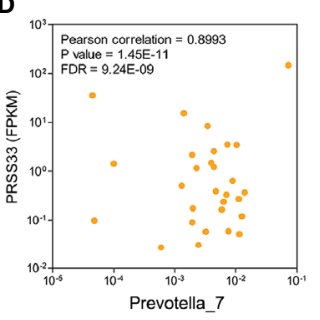

E

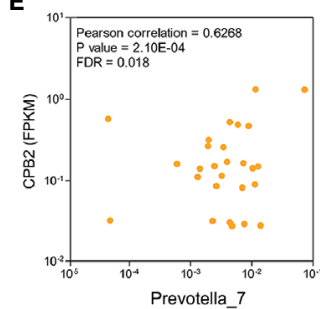

$\mathbf{F}$

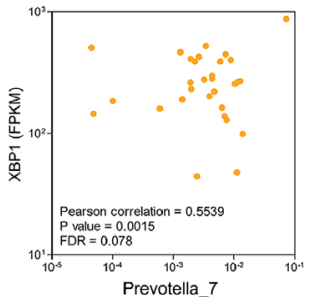

G

CPB2 XBP1

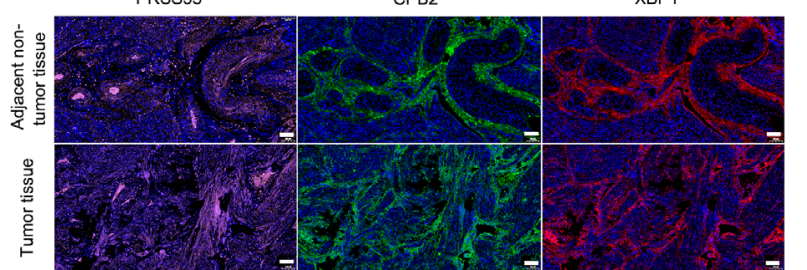

FIGURE 4 | Associations between host endometrial gene expression and Prevotella in patients with EC. (A) Differential expression of Prevotella-associated genes from 30 paired tumor and adjacent nontumor endometrial tissue samples. (B) Integrated analysis of Prevotella-associated genes: microbe-gene correlation dot plot (left panel), $\log _{2} \mathrm{FC}$ value of genes from each patient (middle panel), and functional annotation (right panel). The red line represents the average value. (C) GO enrichment analysis based on the Metascape platform. (D-F) Scatter plots of three typical Prevotella-gene pairs: Prevotella-PRSS33 (D), Prevotella-CPB2 (E) and Prevotella-XBP1 (F). Each point represents a patient. (G) Immunofluorescence showed the expression levels of PRSS33, CPB2 and XBP1 in endometrial tumor and adjacent non-tumor tissues. The white scale bars are $100 \mu \mathrm{m}$.

characteristic (ROC) curve analysis indicated that Prevotella (area under the curve $(\mathrm{AUC})=0.64 ; p=0.047)$, DD (area under the curve $(\mathrm{AUC})=0.73 ; p=0.031$ ), and FDPs $(\mathrm{AUC}=0.79 ; p=$ $0.005)$ were significantly associated with EC samples (Figures 5A-C). In addition, a ROC model in combination with the three could increase the AUC value to $0.86(p=0.001)$ (Figure 5D). The data indicated that these three biomarkers related to fibrin degradation have the potential to predict EC occurrence.

\section{DISCUSSION}

Symptoms of EC, such as abnormal vaginal bleeding, usually present quickly after cancer onset, and most EC patients are diagnosed at an early stage. To elucidate an early disease role for these specific microbes, our study selected women with grade I EC. We provided evidence that the tumor burden is associated with the presence of specific endometrial microbiota, which is distinguished by the enrichment of Pelomonas and Prevotella in patients with EC. Of the two microbes, Prevotella was found to be positively correlated with the increased serum DD and FDPs in the EC group. Further analyses of the host endometrial transcriptome revealed that Prevotella promotes DD and FDPs by influencing the expression of genes related to fibrin degradation. Thus, the results herein on endometrial samples shed light on the gut-transcriptome-uterus axis and may allow for the identification of EC-related biomarkers for clinical prediction.

Compared with the HC group, a significant reduction in alpha diversity was observed in the EC group. Reduced microbial diversity has been reported to lead not only to the outgrowth of a few species and decreased microbial resilience, which is prejudicial to health, but also to chronic diseases, such as cancer (Richard et al., 2018; Liu et al., 2019; Lu et al., 2021). To date, several studies have explored the correlation of microbiota diversity and endometrial disease severity. In one study, Walther-António et al. showed that alpha diversity was increased in the EC group (Walther-António et al., 2016). Other 
A

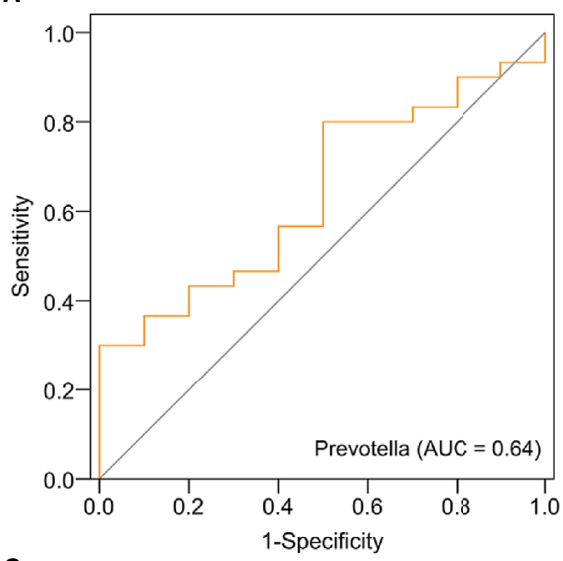

C

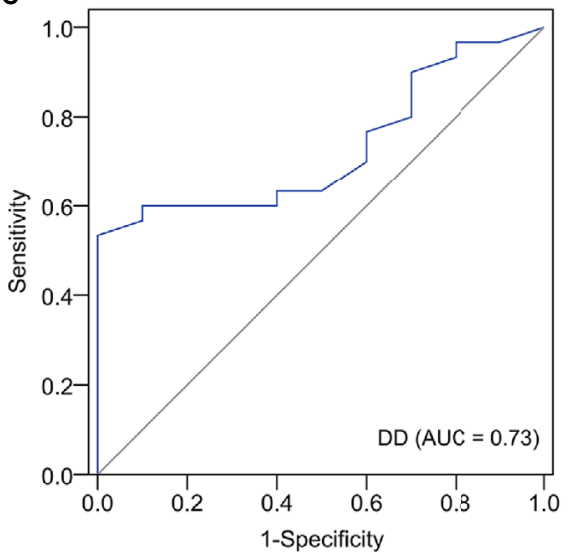

B

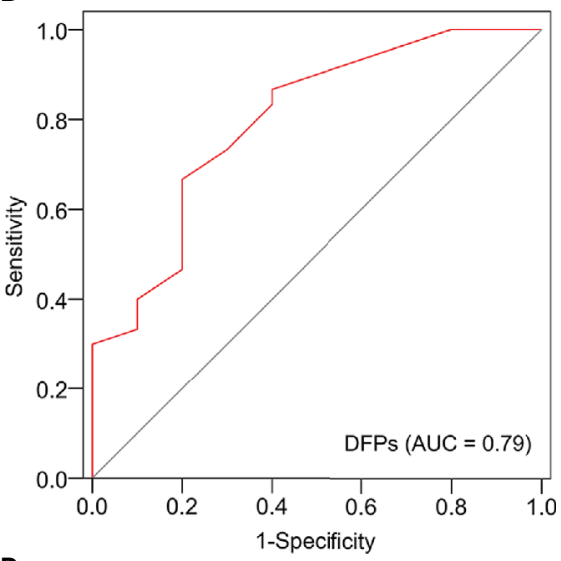

D

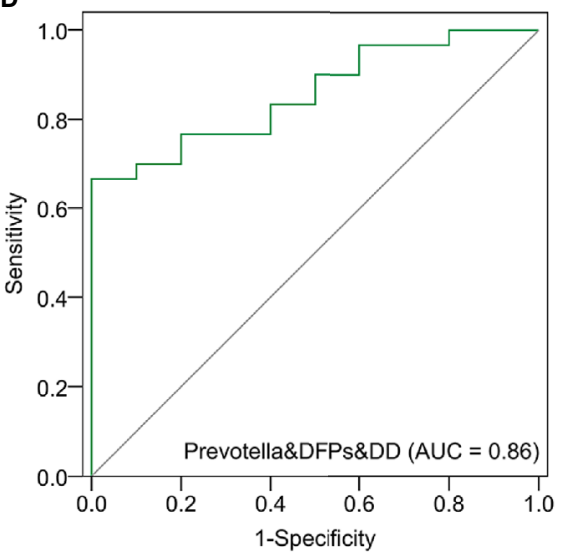

FIGURE 5 | Identification of Prevotella-based markers for the clinical prediction of EC. The receiver operating characteristic (ROC) curve and area under the curve (AUC) were generated to predict the onset of EC based on Prevotella (A), fibrin degradation products (DFPs) (B), D-dimer (DD) (C), and joint modeling of the three factors (D).

evidence has shown that advanced disease severity is associated with decreased endometrial microbiota diversity (Walsh et al., 2019; Lu et al., 2021). The differences could also be attributed to other factors, such as the geographic area, $16 \mathrm{~S}$ rRNA gene target region, and sequencing method (Huang et al., 2018). Nevertheless, our results support the latter point of view.

Compared with previous studies that identified Micrococcus (Lu et al., 2021), Atopobium vaginae and Porphyromonas (Walther-António et al., 2016), or Porphyromas somerae (Walsh et al., 2019) as predictive microbial markers of EC, our results suggest that a higher abundance of Prevotella might favor the development of endometrial tumorigenesis, especially when combined with high serum DD and FDPs. Prevotella, a gramnegative anaerobic bacterial genus, is part of the normal flora within the mouth and vagina (Precup and Vodnar, 2019). Recent studies have suggested a potential role of Prevotella as an intestinal pathobiont related to oral cancer (Karpiński, 2019), colorectal cancer (Flemer et al., 2017; Peng et al., 2020), and lung cancer (Tsay et al., 2018). In particular, this genus has been recognized as a prominent gynecologic and obstetric pathogen positively associated with cervical lesions, including bacterial vaginosis (Marconi et al., 2012), cervical intraepithelial neoplasia (Mitra and MacIntyre, 2020), cervical cancer (Kang et al., 2020), and intrauterine infections (Knoester et al., 2011). Herein, it is interesting to note that Prevotella has an important role in the metabolism of proteins and peptides because many of the members within this genus are actively proteolytic and possess characteristic dipeptidyl peptidase activity (Flint and H., 2014). Based on the correlation of Prevotella with the disease along with its association with the degradation of DD and FDPs, this genus may be involved in the etiology of conditions leading up to EC.

Our results explained the internal reasons for the elevation of DD from the perspective of endometrial microbiota. DD is an end degradation product of fibrin (Kogan et al., 2016), that is considered a diagnostic and prognostic parameter in several malignancies (Fregoni et al., 2012; Ma et al., 2014; Ínal et al., 2015). For example, DD was proven to be directly and positively correlated with EC and its prognosis (Nakamura et al., 2016; Ge et al., 2020). Consistent with previous findings, an elevated DD level was also observed in our study. However, the causes of such an increase are still unclear. Intriguingly, after performing transcriptome analyses, we found altered expression of genes 
related to fibrin degradation in endometrial tumor tissues. Further correlation-based analysis revealed that these genes were attributed to the increased abundance of Prevotella, suggesting that Prevotella might be an important factor that mediates host fibrin degradation.

Vein thrombosis is a common complication in the natural history of malignancies, and patients with cancer have a 4- to 6fold increased risk of developing vein thrombosis compared to patients without cancer (Di Nisio et al., 2010; MacLellan et al., 2012). DD is a signal of the activated coagulation system, and it is currently used as an indicator to assess vein thrombosis probability (Kogan et al., 2016; Khan et al., 2021). Many studies have evaluated the relationship between elevated DD levels and vein thrombosis risk and found that patients with pancreatic, bladder, ovarian and endometrial cancer have the highest incidence of vein thrombosis (Peng et al., 2021). In the present study, we show that Prevotella increased the expression of fibrin degradation-related genes and promoted their protein levels in the EC group, suggesting that Prevotella could be a potential participant in venous thrombosis. Thus, to reduce vein thrombosis in clinical EC, the balance between Prevotella and other microbes should be maintained.

Notably, PRSS33 and XBP1 might be associated with fibrin degradation. Degradation of fibrin is attributed to plasmin, which is one of the most potent serine proteases found in many physiological processes, such as thrombolysis and cancer progression (Deryugina and Quigley, 2012). In our study, the expression of PRSS33 was significantly increased in endometrial tumor tissues, suggesting the functional effect of this protein on fibrin degradation. Although the fibrinolytic activity of PRSS33 was not verified, many studies have described the putative role of serine proteases in hemostasis/thrombosis because plasmin is a nonspecific proteolytic enzyme (Kido et al., 1999; Uesugi et al., 2011; van der Plas et al., 2014; Kakizoe et al., 2016). XBP1 is a transcription factor of the cellular secretory pathway that has been found to be upregulated in hepatic artery thrombosis (Nemes, 2008). Interestingly, a recent study indicated that the overaccumulation of PRSS was accompanied by upregulated XBP1 in a mouse model of chronic pancreatitis. Given the correlation for PRSS33 and XBP1, it is reasonable to speculate that the two proteins may be involved in the elevated DD levels.

Our study has some limitations. First, this is a cross-sectional retrospective study without follow-up data, such as overall survival (OS) and disease-free survival (DFS) data. Hence, we could not determine the classification performance of the Prevotella-fibrin degradation-related genes for discriminating OS or DFS. Second, information about the incidence of venous thrombosis is not provided, and further study regarding this point is suggested. Third, our sample size was relatively small. However, we were still able to identify significant microbiota differences between HCs and ECs and identify specific altered genes that are positively associated with Prevotella and serum DD. Nevertheless, further investigations with an expanded sample size and a multicenter survey are needed to validate the effect of the reported findings.
In summary, we have provided a distinct microbiome signature in patients with EC and healthy controls. The identified Prevotella in the endometrium is associated with the presence of EC, especially when combined with a high concentration of serum DD and FDPs. Prevotella-associated transcripts of host tumors can partly explain how endometrial microbiota promote EC pathogenesis and lead to these aberrant hematologic biomarkers. These findings provide new insights into the connections between the endometrial microbiometranscriptome and biomarker development in the early detection of EC.

\section{DATA AVAILABILITY STATEMENT}

All raw sequences for $16 \mathrm{~S}$ rRNA sequencing were deposited in the NCBI Sequence Read Archive under accession number PRJNA750303. All RNA-seq data generated during this study are available at NCBI GEO under accession number GSE183185. All datasets generated for this study are included in the article/ Supplementary Material.

\section{ETHICS STATEMENT}

This research was approved by the Scientific and Ethical Committee of the Shanghai First Maternity and Infant Hospital affiliated with Tongji University, which is accredited by the National Council on Ethics in Human and Animal Research (KS2033). All human tumor tissues were obtained with written informed consent from patients prior to participation in the study.

\section{AUTHOR CONTRIBUTIONS}

XW and YL designed research. CL, YG, QH, JH, YS, and YL analyzed data. CL and YG wrote the paper. CL, XW, and YL revised the paper. All authors contributed to the article and approved the submitted version.

\section{FUNDING}

This work was supported by the National Natural Science Foundation of China (32070583) and Shanghai Municipal Health Commission (20194Y0156).

\section{SUPPLEMENTARY MATERIAL}

The Supplementary Material for this article can be found online at: https://www.frontiersin.org/articles/10.3389/fcimb.2021. 748558/full\#supplementary-material 


\section{REFERENCES}

Anders, S., Pyl, P. T., and Huber, W. (2015). HTSeq-A Python Framework to Work With High-Throughput Sequencing Data. Bioinformatics 31, 166-169. doi: 10.1093/bioinformatics/btu638

Beral, V., Bull, D., and Reeves, G. (2005). Endometrial Cancer and HormoneReplacement Therapy in the Million Women Study. Lancet 365, 1543-1551. doi: 10.1016/s0140-6736(05)66455-0

Bolger, A. M., Lohse, M., and Usadel, B. (2014). Trimmomatic: A Flexible Trimmer for Illumina Sequence Data. Bioinformatics 30, 2114-2120. doi: 10.1093/bioinformatics/btu170

Bray, F., Ferlay, J., Soerjomataram, I., Siegel, R. L., Torre, L. A., and Jemal, A. (2018). Global Cancer Statistics 2018: GLOBOCAN Estimates of Incidence and Mortality Worldwide for 36 Cancers in 185 Countries. CA Cancer J. Clin. 68, 394-424. doi: 10.3322/caac.21492

Caporaso, J. G., Kuczynski, J., Stombaugh, J., Bittinger, K., Bushman, F. D., Costello, E. K., et al (2010). QIIME Allows Analysis of High-Throughput Community Sequencing Data. Nat. Methods 7, 335-336. doi: 10.1038/ nmeth.f.303

Chen, Y., Guo, J., Shi, D., Fang, D., Chen, C., and Li, L. (2018). Ascitic Bacterial Composition Is Associated With Clinical Outcomes in Cirrhotic Patients With Culture-Negative and Non-Neutrocytic Ascites. Front. Cell. Infect. Microbiol. 8, 420. doi: $10.3389 /$ fcimb.2018.00420

Deryugina, E. I., and Quigley, J. P. (2012). Cell Surface Remodeling by Plasmin: A New Function for an Old Enzyme. J. BioMed. Biotechnol. 2012:564259. doi: $10.1155 / 2012 / 564259$

Di Nisio, M., Ferrante, N., De Tursi, M., Iacobelli, S., Cuccurullo, F., Büller, H. R., et al (2010). Incidental Venous Thromboembolism in Ambulatory Cancer Patients Receiving Chemotherapy. Thromb. Haemost. 104, 1049-1054. doi: $10.1160 /$ th $10-05-0277$

Flemer, B., Lynch, D. B., Brown, J. M., Jeffery, I. B., Ryan, F. J., Claesson, M. J., et al (2017). Tumour-Associated and non-Tumour-Associated Microbiota in Colorectal Cancer. Gut 66, 633-643. doi: 10.1136/gutjnl-2015-309595

Flint, H. J., and Duncan, S. H. (2014). "Bacteroides and Prevotella," in Encyclopedia of Food Microbiology, Batt, C. A., ed. Elsevier, 203-208.

Fregoni, V., Regolo, L., Da Prada, G. A., Zambelli, A., Baiardi, P., Zanini, V., et al (2012). No Correlation Between Plasma D-Dimer Levels and Lymph Node Involvement in Operable Breast Cancer. Breast 21, 220. doi: 10.1016/ j.breast.2011.11.010

Ge, L., Liu, G., Hu, K., Huang, K., Zhang, M., Zhou, J., et al. (2020). A New Risk Index Combining D-Dimer, Fibrinogen, HE4, and CA199 Differentiates Suspecting Endometrial Cancer From Patients With Abnormal Vaginal Bleeding or Discharge. Technol. Cancer Res. Treat. 19, 1533033819901117. doi: $10.1177 / 1533033819901117$

Huang, X., Li, C., Li, F., Zhao, J., Wan, X., and Wang, K. (2018). Cervicovaginal Microbiota Composition Correlates With the Acquisition of High-Risk Human Papillomavirus Types. Int. J. Cancer 143, 621-634. doi: 10.1002/ ijc.31342

Huang, H., Ren, Z., Gao, X., Hu, X., Zhou, Y., Jiang, J., et al (2020). Integrated Analysis of Microbiome and Host Transcriptome Reveals Correlations Between Gut Microbiota and Clinical Outcomes in HBV-Related Hepatocellular Carcinoma. Genome Med. 12, 102. doi: 10.1186/s13073-02000796-5

İnal, T., Anar, C., Polat, G., Ünsal, İ., and Halilçolar, H. (2015). The Prognostic Value of D-Dimer in Lung Cancer. Clin. Respir. J. 9, 305-313. doi: 10.1111/ crj. 12144

Izem, L., Bialkowska, K., Pluskota, E., Das, M., Das, R., and Nieman, M. T. (2021). Plasminogen-Induced Foam Cell Formation by Macrophages Occurs Through a Histone 2B (H2B)-PAR1 Pathway and Requires Integrity of Clathrin-Coated Pits. J. Thromb. Haemost. 19, 941-953. doi: 10.1111/jth.15253

Jonusiene, V., and Sasnauskiene, A. (2021). Notch and Endometrial Cancer. Adv. Exp. Med. Biol. 1287, 47-57. doi: 10.1007/978-3-030-55031-8_4

Kakizoe, Y., Miyasato, Y., Onoue, T., Nakagawa, T., Hayata, M., Uchimura, K., et al (2016). A Serine Protease Inhibitor Attenuates Aldosterone-Induced Kidney Injuries via the Suppression of Plasmin Activity. J. Pharmacol. Sci. 132, 145-153. doi: 10.1016/j.jphs.2016.09.005

Kang, G. U., Jung, D. R., Lee, Y. H., Jeon, S. Y., and Han, H. S. (2020). Dynamics of Fecal Microbiota With and Without Invasive Cervical Cancer and Its
Application in Early Diagnosis. Cancers (Basel) 12, 3800. doi: 10.3390/ cancers 12123800

Karpiński, T. M. (2019). Role of Oral Microbiota in Cancer Development. Microorganisms 7, 20. doi: 10.3390/microorganisms 7010020

Khan, F., Tritschler, T., Kahn, S. R., and Rodger, M. A. (2021). Venous Thromboembolism. Lancet. 398, 64-77. doi: 10.1016/s0140-6736(20)32658-1

Kido, H., Murakami, M., Oba, K., Chen, Y., and Towatari, T. (1999). Cellular Proteinases Trigger the Infectivity of the Influenza A and Sendai Viruses. Mol. Cells 9, 235-244.

Kim, D., Langmead, B., and Salzberg, S. L. (2015). HISAT: A Fast Spliced Aligner With Low Memory Requirements. Nat. Methods 12, 357-360. doi: 10.1038/ nmeth. 3317

Knoester, M., Lashley, L. E., Wessels, E., Oepkes, D., and Kuijper, E. J. (2011). First Report of Atopobium Vaginae Bacteremia With Fetal Loss After Chorionic Villus Sampling. J. Clin. Microbiol. 49, 1684-1686. doi: 10.1128/jcm.01655-10 Kogan, A. E., Mukharyamova, K. S., Bereznikova, A. V., Filatov, V. L., Koshkina, E. V., Bloshchitsyna, M. N., et al (2016). Monoclonal Antibodies With Equal Specificity to D-Dimer and High-Molecular-Weight Fibrin Degradation Products. Blood Coagul. Fibrinolysis 27, 542-550. doi: 10.1097/ mbc. 0000000000000453

Kuźmycz, O., and Staczek, P. (2020). Prospects of NSAIDs Administration as Double-Edged Agents Against Endometrial Cancer and Pathological Species of the Uterine Microbiome. Cancer Biol. Ther. 21, 486-494. doi: 10.1080/ 15384047.2020 .1736483

Li, C., Ao, H., Chen, G., Wang, F., and Li, F. (2019). The Interaction of CDH20 With $\beta$-Catenin Inhibits Cervical Cancer Cell Migration and Invasion via TGF- $\beta /$ Smad/SNAIL Mediated EMT. Front. Oncol. 9, 1481. doi: 10.3389/ fonc. 2019.01481

Lip, G. Y., Chin, B. S., and Blann, A. D. (2002). Cancer and the Prothrombotic State. Lancet Oncol. 3, 27-34. doi: 10.1016/s1470-2045(01)00619-2

Liu, X., Shao, L., Liu, X., Ji, F., Mei, Y., Cheng, Y., et al (2019). Alterations of Gastric Mucosal Microbiota Across Different Stomach Microhabitats in a Cohort of 276 Patients With Gastric Cancer. EBioMedicine 40, 336-348. doi: 10.1016/j.ebiom.2018.12.034

Lu, W., He, F., Lin, Z., Liu, S., Tang, L., Huang, Y., et al (2021). Dysbiosis of the Endometrial Microbiota and Its Association With Inflammatory Cytokines in Endometrial Cancer. Int. J. Cancer 148, 1708-1716. doi: 10.1002/ijc.33428

Ma, X., Li, Y., Zhang, J., Huang, J., and Liu, L. (2014). Prognostic Role of D-Dimer in Patients With Lung Cancer: A Meta-Analysis. Tumour Biol. 35, 2103-2109. doi: 10.1007/s13277-013-1279-9

MacLellan, D. G., Richardson, A., and Stoodley, M. A. (2012). Venous Thromboembolism and Cancer. ANZ J. Surg. 82, 294-298. doi: 10.1111/ j.1445-2197.2012.06047.x

Marconi, C., Cruciani, F., Vitali, B., and Donders, G. G. (2012). Correlation of Atopobium Vaginae Amount With Bacterial Vaginosis Markers. J. Low. Genit. Tract Dis. 16, 127-132. doi: 10.1097/LGT.0b013e31823c79c4

Mitra, A., and MacIntyre, D. A. (2020). The Vaginal Microbiota Associates With the Regression of Untreated Cervical Intraepithelial Neoplasia 2 Lesions. Nat. Commun. 11, 1999. doi: 10.1038/s41467-020-15856-y

Morice, P., Leary, A., Creutzberg, C., Abu-Rustum, N., and Darai, E. (2016). Endometrial Cancer. Lancet 387, 1094-1108. doi: 10.1016/s0140-6736(15) 00130-0

Nakamura, K., Nakayama, K., Ishikawa, M., Katagiri, H., Minamoto, T., Ishibashi, T., et al (2016). High Pretreatment Plasma D-Dimer Levels are Related to Shorter Overall Survival in Endometrial Carcinoma. Eur. J. Obstet. Gynecol. Reprod. Biol. 201, 89-93. doi: 10.1016/j.ejogrb.2016.03.041

Nemes, B. (2008). Some Factors, With an Impact on the Outcome of the Hungarian Liver Transplant Program, With Special Consideration of the Hepatitis C Virus. Magy. Seb. 61, 42-47. doi: 10.1556/MaSeb.61.2008.1.9

O'Keefe, S. J. (2016). Diet, Microorganisms and Their Metabolites, and Colon Cancer. Nat. Rev. Gastroenterol. Hepatol. 13, 691-706. doi: 10.1038/ nrgastro.2016.165

Peng, Z., Cheng, S., Kou, Y., Wang, Z., Jin, R., Hu, H., et al (2020). The Gut Microbiome Is Associated With Clinical Response to Anti-PD-1/PD-L1 Immunotherapy in Gastrointestinal Cancer. Cancer Immunol. Res. 8, 12511261. doi: 10.1158/2326-6066.cir-19-1014

Peng, M., Yang, S., Li, G., Zhang, T., Qin, X., Shi, C., et al (2021). Solid Tumor Complicated With Venous Thromboembolism: A 10-Year Retrospective 
Cross-Sectional Study. Clin. Appl. Thromb. Hemost. 27, 1076029620975484. doi: $10.1177 / 1076029620975484$

Precup, G., and Vodnar, D. C. (2019). Gut Prevotella as a Possible Biomarker of Diet and Its Eubiotic Versus Dysbiotic Roles: A Comprehensive Literature Review. Br. J. Nutr. 122, 131-140. doi: 10.1017/s0007114519000680

Reyon, D., Tsai, S. Q., Khayter, C., Foden, J. A., Sander, J. D., and Joung, J. K. (2012). FLASH Assembly of TALENs for High-Throughput Genome Editing. Nat. Biotechnol. 30, 460-465. doi: 10.1038/nbt.2170

Richard, M. L., Liguori, G., Lamas, B., Brandi, G., da Costa, G., Hoffmann, T. W., et al (2018). Mucosa-Associated Microbiota Dysbiosis in Colitis Associated Cancer. Gut Microbes 9, 131-142. doi: 10.1080/19490976.2017.1379637

Roberts, A., Trapnell, C., Donaghey, J., Rinn, J. L., and Pachter, L. (2011). Improving RNA-Seq Expression Estimates by Correcting for Fragment Bias. Genome Biol. 12, R22. doi: 10.1186/gb-2011-12-3-r22

Rognes, T., Flouri, T., Nichols, B., Quince, C., and Mahé, F. (2016). VSEARCH: A Versatile Open Source Tool for Metagenomics. PeerJ 4, e2584. doi: 10.7717/ peer. 2584

Segata, N., Izard, J., Waldron, L., Gevers, D., Miropolsky, L., Garrett, W. S., et al (2011). Metagenomic Biomarker Discovery and Explanation. Genome Biol. 12, R60. doi: 10.1186/gb-2011-12-6-r60

Stelloo, S., Linder, S., Nevedomskaya, E., Valle-Encinas, E., de Rink, I., Wessels, L. F. A., et al (2020). Androgen Modulation of XBP1 is Functionally Driving Part of the AR Transcriptional Program. Endocr. Relat. Cancer 27, 67-79. doi: 10.1530/erc-19-0181

Trapnell, C., Williams, B. A., Pertea, G., Mortazavi, A., Kwan, G., van Baren, M. J., et al (2010). Transcript Assembly and Quantification by RNA-Seq Reveals Unannotated Transcripts and Isoform Switching During Cell Differentiation. Nat. Biotechnol. 28, 511-515. doi: 10.1038/nbt.1621

Tsay, J. J., Wu, B. G., Badri, M. H., Clemente, J. C., Shen, N., Meyn, P., et al (2018). Airway Microbiota Is Associated With Upregulation of the PI3K Pathway in Lung Cancer. Am. J. Respir. Crit. Care Med. 198, 1188-1198. doi: 10.1164/ rccm.201710-2118OC

Uesugi, Y., Usuki, H., Iwabuchi, M., and Hatanaka, T. (2011). Highly Potent Fibrinolytic Serine Protease From Streptomyces. Enzyme Microb. Technol. 48, 7-12. doi: 10.1016/j.enzmictec.2010.08.003

van der Plas, M. J., Andersen, A. S., Nazir, S., van Tilburg, N. H., Oestergaard, P. R., Krogfelt, K. A., et al (2014). A Novel Serine Protease Secreted by Medicinal
Maggots Enhances Plasminogen Activator-Induced Fibrinolysis. PloS One 9, e92096. doi: 10.1371/journal.pone.0092096

Walsh, D. M., Hokenstad, A. N., Chen, J., Sung, J., Jenkins, G. D., Chia, N., et al (2019). Postmenopause as a Key Factor in the Composition of the Endometrial Cancer Microbiome (ECbiome). Sci. Rep. 9, 19213. doi: 10.1038/s41598-01955720-8

Walther-António, M. R., Chen, J., Multinu, F., Hokenstad, A., Distad, T. J., Cheek, E. H., et al (2016). Potential Contribution of the Uterine Microbiome in the Development of Endometrial Cancer. Genome Med. 8, 122. doi: 10.1186/ s13073-016-0368-y

Wang, Q., Garrity, G. M., Tiedje, J. M., and Cole, J. R. (2007). Naive Bayesian Classifier for Rapid Assignment of rRNA Sequences Into the New Bacterial Taxonomy. Appl. Environ. Microbiol. 73, 5261-5267. doi: 10.1128/aem.00062-07

Yang, Y., Misra, B. B., Liang, L., Bi, D., Weng, W., Wu, W., et al (2019). Integrated Microbiome and Metabolome Analysis Reveals a Novel Interplay Between Commensal Bacteria and Metabolites in Colorectal Cancer. Theranostics 9, 4101-4114. doi: 10.7150/thno.35186

Zaura, E., Keijser, B. J., Huse, S. M., and Crielaard, W. (2009). Defining the Healthy "Core Microbiome" of Oral Microbial Communities. BMC Microbiol. 9, 259. doi: 10.1186/1471-2180-9-259

Conflict of Interest: The authors declare that the research was conducted in the absence of any commercial or financial relationships that could be construed as a potential conflict of interest.

Publisher's Note: All claims expressed in this article are solely those of the authors and do not necessarily represent those of their affiliated organizations, or those of the publisher, the editors and the reviewers. Any product that may be evaluated in this article, or claim that may be made by its manufacturer, is not guaranteed or endorsed by the publisher.

Copyright $\odot 2021 \mathrm{Li}, \mathrm{Gu}, \mathrm{He}$, Huang, Song, Wan and Li. This is an open-access article distributed under the terms of the Creative Commons Attribution License (CC BY). The use, distribution or reproduction in other forums is permitted, provided the original author(s) and the copyright owner(s) are credited and that the original publication in this journal is cited, in accordance with accepted academic practice. No use, distribution or reproduction is permitted which does not comply with these terms. 